\title{
Reutilización de catéteres de angiografía en una Unidad de Hemodinamia de un hospital del sector público-Chile
}

\author{
Andrés Scherson B., MSc y Humberto Dighero T., MD
}

\section{Angiographic catheter reuse at the Hemodynamic Department of a public hospital in Chile}

This article presents the results of a study conducted in 421 patients who underwent invasive procedures utilizing catheters for diagnosis and treatment at the Hemodynamic Department of a Public Hospital during the period between July and December 2003. The goal of this study was to determine the existence of risks in the reuse of disposable angiographic catheters. This study was designed as an observational prospective cohort follow up. From the sample studied, 63.4\% were males with average age of 64 years. Co-morbidities were diabetes mellitus and obesity in 27 and $24 \%$ respectively. Eighty two percent of the procedures were diagnostic and reused catheters were used in $74 \%$ of all procedures. Local infection or systemic pyrogenic reaction was observed in $4.3 \%$ patients, however there were no significant differences in any of the variables studied, between the group in which reused catheters were used and the group with new catheters $(p<0.05)$.

Key words: Angiography, angiographic catheter, reuse, temporary bacteremia, pyrogenic reactions, non systemic infections.

Palabras claves: Angiografía, catéter de angiografía, reutilización, bacteriemia transitoria, reacciones pirogénicas, infecciones no-sistémicas.

\section{Introducción}

$\mathbf{L}$ as técnicas de angiografía coronaria más frecuentemente utilizadas consisten en la inserción percutánea de un catéter a través de la punción de una arteria periférica (femoral común o radial). Cuando se toman las debidas precauciones la coronariografía tiene escaso peligro. Por la naturaleza del problema que se investiga es posible que el paciente presente dolor anginoso y raramente un infarto al miocardio. Tratándose de un examen en que se introducen catéteres al sistema arterial pueden ocurrir complicaciones de tipo embólico o complicaciones locales en el sitio de la punción arterial.

Los catéteres utilizados se presentan estériles, con diversas formas y tamaños. Son radio-opacos y cada tipo puede tener diferentes curvas y calibres, entre $4 \mathrm{~F}$ y $8 \mathrm{~F}$ y están catalogados como de uso único.

De una revisión sistemática de la literatura médica pueden extraerse algunos datos de las complicaciones del procedimiento de cateterización cardíaca en los últimos 10 años. Un total de 7.412 cateterizaciones cardíacas diagnósticas consecutivas fueron realizadas entre enero 1990 y diciembre 2000 y fueron registradas en forma prospectiva. En este estudio hubo un total de 63 complicaciones, de las cuales 40 fueron menores y 23 mayores. La tasa general de complicacio- nes fue de $0,8 \%$, con una tasa de mortalidad de $0 \%$. Un análisis univariado mostró una menor tasa general de complicaciones asociadas a médicos senior $(>$ de 500 angiografías coronarias realizadas (OR 0,$50 ; p=0,04)$, tamaño menor del catéter $(\mathrm{OR}=2,6 ; \mathrm{p}=0,0004)$, y una mayor tasa en pacientes con cateterización cardíaca izquierda que derecha $(\mathrm{OR}=2,62 ; \mathrm{p}=0,003)^{1-4}$. Los únicos factores de riesgo predictores para complicaciones mayores en la angiografía coronaria fueron el tamaño del catéter y el peso corporal ${ }^{10,12,13}$.

Con respecto a la experiencia local, en un estudio presentado en el X Congreso Chileno de Infección Intrahospitalaria 2001 (Puerto Varas), se presentó la experiencia de un centro de salud privado en el cual se realizó seguimiento a 1.318 pacientes sometidos a procedimientos hemodinámicas, diagnósticos y terapéuticos durante el período 1999-2000. La incidencia de infecciones locales fue $2,9 \%$ y de infecciones sistémicas $0 \%$. Las complicaciones infecciosas estuvieron relacionadas tanto a uso de catéteres nuevos como reutilizados ${ }^{14}$.

Todos los artículos publicados coinciden en que con un protocolo documentado de reutilización no existiría diferencia entre el uso de catéteres nuevos y reutilizados ${ }^{4-10}$.

No hay datos publicados en la literatura médica sobre la prevalencia de infecciones y/o complicacio-
Establecimiento donde se realizó el trabajo: Hospital San Juan de Dios de Santiago

Recibido: 26-07-04 Aceptado: 05-12-05

Correspondencia a: Andrés Scherson B. ascherson@ hotmail.com 
nes en pacientes sometidos al procedimiento de hemodinamia en los hospitales públicos en Chile. Dado que éste es un procedimiento frecuente en muchas de estas instituciones, y que por costos se reutilizan catéteres diseñados y fabricados para un solo uso, es relevante realizar el presente estudio en un hospital público.

\section{Material y Método}

Se realizó un estudio epidemiológico de tipo analítico observacional de cohortes prospectivas ${ }^{11}$ cuyo objetivo general fue conocer las consecuencias de la reutilización de catéteres de angiografía catalogados como de uso único, en la unidad de hemodinamia de un hospital público.

\section{Tabla 1. Datos globales de los procedimientos realizados en hemodinamia} durante el período julio-diciembre 2003

\begin{tabular}{|c|c|c|c|c|}
\hline & \multicolumn{2}{|c|}{$\begin{array}{l}\text { Catéter nuevo } \\
\quad(n=108)\end{array}$} & \multicolumn{2}{|c|}{$\begin{array}{l}\text { Catéter reutilizado } \\
\qquad(n=313)\end{array}$} \\
\hline & & (\%) & n & (\%) \\
\hline Sexo masculino & 73 & $(67,6)$ & 194 & $(61,9)$ \\
\hline Sexo femenino & 35 & $(32,4)$ & 119 & $(38,0)$ \\
\hline Procedimientos diagnósticos & 88 & $(81,4)$ & 257 & $(82,1)$ \\
\hline Procedimientos terapéuticos & 20 & $(18,5)$ & 56 & $(17,9)$ \\
\hline Obesidad & 16 & $(14,8)$ & 86 & $(27,4)$ \\
\hline Diabetes mellitus & 31 & $(28,7)$ & 84 & $(26,8)$ \\
\hline $\begin{array}{l}\text { Tiempo promedio de procedimiento } \\
\text { diagnóstico (min) }\end{array}$ & \multicolumn{2}{|c|}{23,7} & \multicolumn{2}{|c|}{22,07} \\
\hline $\begin{array}{l}\text { Tiempo promedio de procedimiento } \\
\text { terapéutico (min) }\end{array}$ & \multicolumn{2}{|c|}{61} & \multicolumn{2}{|c|}{60,8} \\
\hline
\end{tabular}

Los catéteres fueron reprocesados de acuerdo a las recomendaciones de reproceso de catéteres de hemodinamia del Ministerio de Salud ${ }^{15}$ y esterilizados en óxido de etileno.

Los participantes en el estudio fueron todos los pacientes que acudieron a la Unidad de Hemodinamia del Hospital San Juan de Dios durante el período juliodiciembre del 2003 y que fueron sometidos a procedimientos invasores utilizando catéteres con fines diagnósticos y terapéuticos en enfermedades cardiovasculares.

Los datos de cada paciente fueron obtenidos del libro de registro del pabellón de hemodinamia, así como del registro de vigilancia epidemiológica, hoja de registro de complicaciones y revisión de fichas clínicas.
Se excluyeron del estudio todos aquellos pacientes en los cuales no se consignó si los catéteres utilizados en el procedimiento eran nuevos o usados.

Todos los datos fueron almacenados en una base de datos (Microsoft Excel 2000 que después fue copiada en Minitab 2003 para efectos de cálculos). El análisis estadístico se hizo mediante tablas de contingencia, cálculo de $\chi^{2}$ (con corrección de Yates). Las variables de tipo continuo se analizaron mediante el método t de Student (análisis de la varianza).

Se minimizaron los sesgos con un buen diseño que incluyó la planificación detallada de la constitución de la cohorte y los mecanismos de seguimiento además de los instrumentos de captura de información.

Se determinó el tamaño de muestra bajo los siguientes criterios: la prevalencia de complicaciones en el procedimiento es de $2 \%{ }^{4}$ y la probabilidad de un error tipo Il $(\alpha)=0,05 \%$. Dado que no hay publicaciones nacionales de incidencia trabajamos con un supuesto de 4 a $5 \%$ por lo que tomamos precisión máxima de $3 \%$ y una potencia de la prueba de $60 \%$.

Se confeccionaron cohortes de pacientes en cuyos procedimientos fueron utilizados catéteres de angiografía de uso único y aquellos en que los catéteres eran reprocesados (más de un uso).

Se resguardaron los aspectos éticos de la investigación. El estudio fue autorizado por el Comité de Ética Científica del hospital. Los datos incluidos en el estudio son globales y no se cita a pacientes en particular.

\section{Resultados}

Se vigilaron entre julio y diciembre del 2003 un total de 421 procedimientos correspondiendo $63,4 \%$ (267) a pacientes de sexo masculino y $36,6 \%$ (154) a mujeres. (Tabla 1).

El 81,9\% (345) de los procedimientos realizados tuvo carácter diagnóstico y $18,1 \%$ (78) correspondieron a procedimientos terapéuticos.

Del total, $25,6 \%$ (108) se realizaron con catéteres nuevos y 74,3\% (313) con catéteres reutilizados. (Tabla 1). El promedio de edad fue de 60,27 (DS 10,26).

Se observó una letalidad de $2,1 \%$; sin embargo, sólo 3 de estos pacientes fallecieron en el pabellón de hemodinamia $(0,7 \%)$ (Tabla 2$)$.

Las complicaciones no infecciosas, incluyendo la reacción a pirógenos tuvieron una incidencia de 3,3\% y las catalogadas como de tipo infeccioso 1,2\% (Tabla 2). Sin embargo, en $100 \%$ de ellas los hemocultivos fueron negativos.

La Tabla 3 muestra el análisis estadístico univariado mediante RR, PSE y $\chi^{2}$ para distinguir las variables predictoras (condición del catéter, sexo, diabetes 
mellitus y obesidad) que hubieran podido influir en la presencia de las complicaciones e infecciones, considerando como una variable significativa aquella que alcanza un valor de $p$ igual o menor a 0,05 . No hubo diferencia significativa con respecto a la presencia de complicaciones entre emplear catéter nuevo o reutilizado (RR 1,15; p 0,72). Tampoco se observaron diferencias significativas por género $(\mathrm{p} 0,66)$ ni con respecto a diabetes mellitus ( $p 0,24)$. La obesidad no determinó diferencias significativas en la incidencia de complicaciones infecciosas y no infecciosas en esta

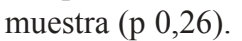

No hubo diferencia significativa en los tiempos de los procedimientos diagnósticos y terapéuticos realizados con catéter nuevo respecto a los realizados con catéteres reutilizados (Tabla 4). Tanto en los procedimientos diagnósticos como terapéuticos se verificó que existiera estabilidad del proceso (I-MR) y que hubiera una distribución normal (mediante test de normalidad de Anderson-Darling).

\section{Discusión}

Para reducir costos, la reutilización de catéteres de hemodinamia es común en muchos países, aunque no ha sido estudiada en forma sistemática en el pasado. Es habitual para equipos quirúrgicos y algunos otros artículos el ser esterilizados y reutilizados en forma segura. Las preocupaciones relacionadas al reempleo de catéteres de hemodinamia incluyen el riesgo de infecciones bacterianas y virales, el riesgo de reacciones a pirógenos ${ }^{16}$, implicancias médico-legales y éticas, y el argumento que cualquier ahorro en costos pudiera resultar en un aumento de las complicaciones. Con una limpieza y esterilización adecuada, y asegurando la permeabilidad del lumen del catéter, el riesgo de infecciones bacterianas debido la reutilización de catéteres pareciera no aumentar. Aunque está documentado que residuos de la re -esterilización quedan en los catéteres, esta parece ser una concepción teórica, ya que un adecuado protocolo de limpieza y esterilización hacen que los riesgos de reacciones pirogénicas con catéteres re-utilizados sean similares a los observados con catéteres nuevos, en el entendido que el reempleo de catéteres de angiografía es éticamente lícito sólo si la incidencia de efectos adversos es igual o menor que la asociada con el uso de catéteres nuevos.

Los resultados de este trabajo muestran que no hay diferencia respecto a los efectos adversos de la reutilización de los catéteres de hemodinamia.

Uno de los factores que inciden en complicaciones del procedimiento de angiografía es la experiencia de los profesionales intervensionistas ${ }^{17}$. En la Unidad de Hemodinamia de hospital donde se efectuó el estudio, todos los profesionales que realizaron los procedimientos durante el período en estudio tenían más de 500 horas de experiencia en este procedimiento.

La tasa de complicaciones no infecciosas en nuestra institución $(4,3 \%)$ está sobre lo que se encuentra en

Tabla 2. Incidencia de complicaciones, reacción a pirógenos, infecciones y fallecimientos

\begin{tabular}{|lcccc|}
$\begin{array}{l}\text { Mes del } \\
\text { procedimiento }\end{array}$ & Complicaciones & $\begin{array}{l}\text { Reacción a } \\
\text { pirógenos }\end{array}$ & Infecciones & Fallecidos \\
\hline Julio & 6 & - & 3 & 3 \\
\hline Agosto & 2 & - & - & 2 \\
\hline Septiembre & 2 & - & 1 & 2 \\
\hline Octubre & 1 & - & - & - \\
\hline Noviembre & 1 & 1 & - & 2 \\
\hline Diciembre & - & 1 & 1 & - \\
\hline Total & 12 & 2 & 5 & 9 \\
\hline Porcentaje (\%) & 2,85 & 0,095 & 1,2 & 2,1 \\
\hline
\end{tabular}

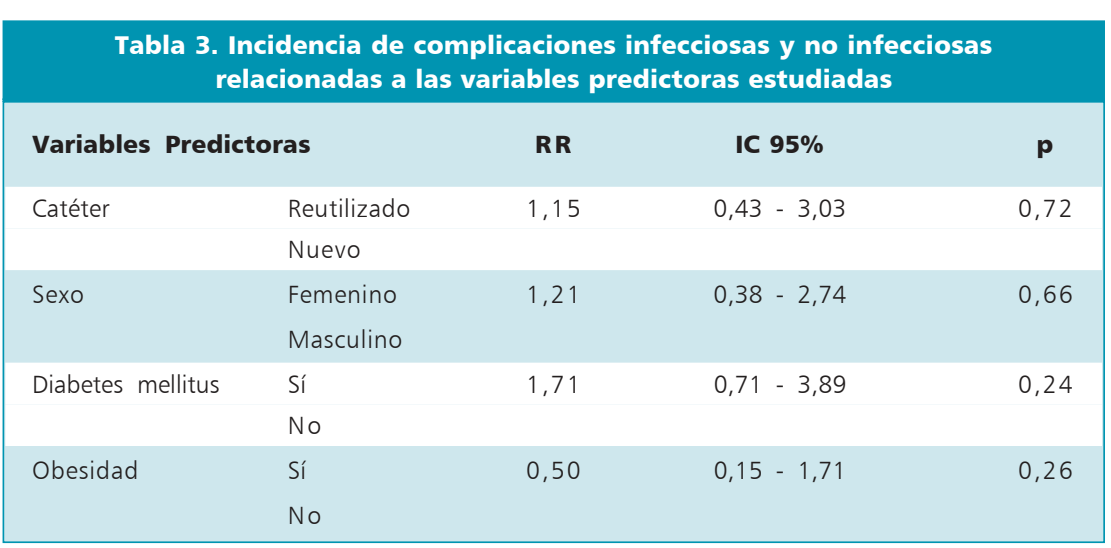

\begin{tabular}{|l|llll|}
\hline \multicolumn{5}{|c|}{ Tabla 4. Tiempo de procedimientos diagnósticos y terapéuticos } \\
en relación a condición del catéter
\end{tabular}


la literatura internacional, la relevancia clínica de esta diferencia no es discutida en este trabajo.

En ninguna de las variables predictoras estudiadas se encontró una asociación con complicaciones infecciosas y no infecciosas, a diferencia de los hallazgos señalados en la literatura científica que asignan al peso corporal 1,4 veces mayor riesgo de complicaciones por cada $10 \mathrm{~kg}$ de peso con significación estadística.

Una limitación del estudio fue el reducido tamaño de muestra (potencia de 60\%) debido a que se excluyó a aquellos pacientes en los cuales no se consignó la condición del catéter (nuevo/reutilizado). También fue limitante el tiempo disponible para el estudio.

En conclusión, no se encontraron diferencias significativas al realizarse procedimientos hemodinámicas con catéter nuevo o reutilizado.

De acuerdo a estos hallazgos preliminares y a las limitaciones del estudio se recomendaría ampliar el estudio a un protocolo multicéntrico.

Debido al creciente número de procedimientos de hemodinamia que se realiza en instituciones de salud y a las bajas tasas de complicaciones observadas al emplear catéteres reutilizados cuando se aplicaron estrictos protocolos de reprocesamiento según la literatura médica extranjera, se podría recomendar el aplicar estos protocolos con vigilancia epidemiológica y supervisión enmarcadas en un programa de calidad continua.

\section{Agradecimientos}

Mis más sinceros agradecimientos al Director y Sub Director del Hospital San Juan de Dios, Rafael Ron- danelly I. y Marcos Sepúlveda C., respectivamente, así como a todo el personal de la Unidad de Hemodinamia del citado hospital, sin cuyo valioso apoyo no hubiera sido posible concretar esta investigación. Especial mención merece la Enfermera de Infección Intrahospitalaria del establecimiento, Sra Carmen Laytte V.

\section{Resumen}

Se presentan los resultados de un estudio realizado en 421 pacientes que se sometieron a procedimientos invasores utilizando catéteres con fines diagnósticos y terapéuticos en la Unidad de Hemodinamia de un hospital público en el período julio-diciembre del año 2003. El objetivo de la investigación fue conocer si existen riesgos en la re-utilización de catéteres de angiografía empleados en dicha unidad y que son catalogados como de uso único. Para este fin se seleccionó como metodología de investigación la de estudio epidemiológico de tipo analítico observacional de cohortes prospectivas. De la población estudiada, $63,4 \%$ correspondió a población masculina con edad promedio de 64 años. Del total de los pacientes $27 \%$ presentaban diabetes mellitus y $24 \%$ obesidad. El $82 \%$ de los procedimientos tenía fines diagnósticos y 74\% fueron realizados con catéteres reutilizados.

Se encontró $4,3 \%$ de complicaciones infecciosas locales o reacciones a pirógenos; sin embargo, no se encontró diferencia significativa entre el grupo que fue intervenido con catéteres de primer uso respecto a los que utilizaron catéteres antes usados $(\mathrm{p}<0,05)$ en ninguna de las variables estudiadas.

\section{Referencias}

1.- Weinstein R A. Adding insult to injury: Device related infections. $38^{\text {th }}$ Annual Meeting of the Infectious Diseases Society of America, 2000

2.- Bentolila P, Jacob R, Roberge F. Effects of re-use on the physical characteristics of angiographic catheters. J Med Eng Technol 1990; 14: 254-9, Abstract PMID: 2283675 (PubMed- indexed for MEDLINE).

3.- Abreu E L, Haire D M, Malchesky P S, WolfBloom D F, Cornhill J F. Development of a program model to evaluate the potencial for reuse single-use medical devices: results of a pilot test study. Biomed Instrum Technol 2002; 36: 389-404.

4.- American College of Cardiology/Society for Cardiac Angiography and Interventions
Clinical Expert Consensus Document on Cardiac Catheterization Laboratory Standards 2001; 37: 2172-211.

5.- Mak K H, Eisenberg M J, Eccleston D S, Cornhill J F, Topol E J. Reuse of coronary angioplasty equipment: technical and clinical issues. Am Heart J 1996; 131: 624-30.

6.- Browne K F, Maldonado R, Telatnik M, Vlietstra R E, Brenner A S. Inicial experience with reuse of coronary angioplasty catheters in the United States. J Am Coll Cardiol 1997; 30: 1735-40.

7.- Zubaid M, Thomas C S, Salman H, AlRashdan I, Hayat N, Habashi A, et al. A randomized study of the safety and efficacy of reused angioplasty balloon catheters. Indian Heart J 2001; 53: 167-71.

8.- Grimandi G, Sellal O, Grimaldi F, Crochet D. Risks of reusing coronary angioplasty catheters: results of an experimental study. Cathet Cardiovasc Diagn 1996; 38: 123-30; discussion 131-2.

9.- The reuse of single-use catheters, Montreal: Conseil d"valuation des technologies de la sant, du Quebec, 1993. The reuse of singleuse Medical Devices, Canadian Hospital Association.

10.- Comis J. Reusing single-use cardiac catheters. Dimens Health Serv 1991; 68: 8-9, 34-5. Review.

11. Lazcano-Ponce E, Fernándes E, Salazar E, Hernández Ávila M. Estudios de cohorte. Metodología, sesgos y aplicación. Salud Pública de México 2000; 42: 230-41.

12.- Shaw JP, Eisenberg MJ, Azoullay A, Nguyen N. Reuse of catheters for percutaneous transluminal coronary angioplasty: effects on procedure time and clinical outcomes. 
Catheter Cardiovasc Interv 1999; 48: 54-60.

13.- Luijt D S, Schirm J, Savelkoul P H,

Hoekstra A. Risk of infection by

reprocessed and resterilized virus-

contaminated catheters; an in-vitro study.

Eur Heart J 2001; 22: 378-84.

14.- Muñoz C P. Reutilización de catéteres de hemodinamia, X Congreso Chileno de Infecciones Intrahospitalarias y
Epidemiología Hospitalaria, Puerto Varas 2001; Conferencia Plenaria.

15.- Ministerio de Salud. Normas Técnicas sobre Esterilización y Desinfección de Elementos Clínicos 2001: 126.

16.- Ajeka S, Malamam A, Da Silva P A. Pirogenia em cateterismo cardíaco: Deteccao da causa e erradicacao, pela padronizacao do reprocesamiento de materiais, Temas Livres Enfermagen, 201, Belho Horizonte, 2002.

17.- Ammann P, Brunner-La Rocca H P, Anghern W, Roelli H, Sagmeister M. Procedural complications following diagnostic coronary angiography are related to the operator"s experience and the catheter size. Catheter Cardiovasc Interv 2003; 59: 13-8. 